\title{
Clinical applications of occlusion principles in implantology - Narrative review
}

\section{Oliveira A, Bessa A, Dias F}

\section{Corresponding author:}

Ana Oliveira

ana.cb.oliveira@hotmail.com

\section{ABSTRACT:}

Purpose: The objective of this paper is to review concepts of this type of occlusion and provide clinical guidelines based on the currently available literature. Materials and Methods: A literature review was conducted in MEDLINE and PubMed since September 2015 to January 2016.

Results: The database searches resulted in 1481 potentially relevant articles. After inclusion/exclusion criteria 41 articles were considered.

Conclusions: Based on the literature reviewed it can be concluded that the objective of implant-protected occlusion is to minimize occlusal overload on the implant / bone interface and on prostheses keeping the masticatory loads within physiological limits, providing stability and longevity to rehabilitation treatments with implants.

\section{KEYWORDS}

Occlusion, implants, implant-supported prosthesis, implant protected occlusion. 


\section{INTRODUCTION}

The technological advances in dental implants osseointegration facilitate the long-term results of fixed prosthetic treatment. ${ }^{1-4}$ The modification of dental implants design was proposed to improve the osseointegration process. ${ }^{2,3}$ Nevertheless, it remains a risk of implant failure as result of peri-implant bone loss. This process can occur due to biological or mechanical causes. ${ }^{1,4-7}$ Occlusal overload appears to be an important mechanical factor. ${ }^{1}$ Improper occlusion, associated with inappropriate muscular forces, promote a non-acceptable biologically stress around the implant components and bone implant interface causing overload implant, which leads to stress fractures, fatigue, cement breakdown and screw loosening. ${ }^{3,}$ 5, 6, 8-14 Therefore, conventional occlusal concepts must be adapted in prosthetic treatments supported by dental implants. The ideal implant protected occlusion provides the longevity of both implant as well as the prosthesis. ${ }^{3,15-20}$ The objective of this paper is to review concepts of implant protected occlusion and provide clinical guidelines based on the currently available literature.

\section{MATERIALS AND METHODS}

A literature review was conducted in MEDLINE and PubMed since September 2015 to January 2016, being used the following keywords: "implant-protected occlusion", "occlusion and implantology" and "implant-supported dentures." It has been found 1481 articles in English.

\section{RESULTS}

The database searches resulted in 1481 potentially relevant articles. All the items that did not have full text where excluded, studies with non-significant samples and studies whose empirical classical concepts were admitted. At the end 41 articles were evaluated: 22 review articles, 16 randomized controlled trials and 3 case reports.

\section{DISCUSSION}

The biomechanical control of occlusal factors has been considered in determining the prognosis of dental implants and clinical success and longevity of the implant-supported rehabilitation treatments. ${ }^{21,22}$

The existence of implant overload is directly related to the occurrence of mechanical complications or treatment failure after restore the patient function. The most commonly complications or failures reported and observed in clinical practice are the fracture or loosening of the screw, fracture of the clinical crown, fracture of the prosthesis and bone loss at the level of the crest with consequent implant loosening. ${ }^{18}$

This literature review considers the occlusal factors that are critical to do rehabilitations with fixed implant prosthetic treatments. Thus, the principles can be applied to minimizing overload at the interface between implant and bone tissue while maintaining the occlusal load over the implant within a physiological limit.

The first factor considered is the physiological difference between natural teeth and osseointegrated implants (this difference is not mostly considered at the beginning of treatment and may result in overload and failure of dental rehabilitation). When this difference is not considered enough at the beginning of the treatment this may result in overload and failure of the dental rehabilitation. 5, 7, 19, 23 The major difference between natural teeth and dental implants is the presence of periodontal ligament (PDL). It is known that implants have a minor resiliency compared to natural teeth due to the absence of PDL. ${ }^{5,8,23}$ The mean value of axial displacement of teeth in the socket is 25 $100 \mu \mathrm{m}$, while that of osseointegrated implants has been reported approximately 3-5 $\mu \mathrm{m}$. $^{23-25}$

The PDL has mechanic receptors that confer tactile sensitivity, protecting teeth and periodontum of occlusal overloading. ${ }^{21,23}$ Dental fulcrum is located in the apical third of the teeth, where charges are absorbed and stress is distributed at the bone tissue leading the non-axial forces towards functional axis of the teeth. ${ }^{18,20,24}$ The clinical signs of overloading include PDL thickening, mobility, dental wear facets and pain. ${ }^{24}$

Dental implants are unprotected of excessive occlusal forces because they do not have this natural buffer. The proprioceptive mechanism is given by osseoperception. ${ }^{18,}$ 23, 25 Therefore crestal bone around the implant acts as the fulcrum when a force is applied inducing a greater susceptibility to bone loss in the peri-implant tissues. ${ }^{26}$ In case of occlusal overload, one or more components of the implant system can fail, for instance, fracture of the implant itself, loosening or fracture of connecting screws or abutment screws, loosening or excessive wear of structural components in overdentures and excessive wear or fracture of suprastructural porcelain or acrylic teeth. ${ }^{18,23,27}$ When partially edentulous dental arches are rehabilitated, the stability of remaining teeth must be evaluated. During the contacts of mild to moderate intensity implant-supported prosthesis should be in slight infra-occlusion to compensate the biomechanical differences between natural teeth and implants when strong contact happens. That means that any mobility in natural teeth induces an additional charge to implant-supported prostheses. Thus, dental interferences must be diagnosed and corrected in order to obtain a stable occlusion. ${ }^{18}$

Bone quality is another important factor for long-term success of prosthetic rehabilitation. Loss of marginal bone support is related to bone quality: the lower the bone density, greater is the risk of peri-implant bone loss after functional loading. 8, 10, 19, 24, 28 To avoid this problem it was proposed by some author's the progressive loading procedure. ${ }^{18,23,24}$ This technique promotes a good integration of the implant in the bone, increasing its density and decrease bone loss at the crestal level. ${ }^{19}$

Immediate loading provides the functional and psychosocial patient rehabilitation. However, some studies suggested a possibility of bone resorption around implants with $180 \mu \mathrm{m}$ of excess suprastructure height, even in the absence of peri-implant inflammation in cases of immediate loading. ${ }^{11,29,30} \mathrm{It}$ is therefore important that the rehabilitation of these cases is performed with restricted parameters which include adequate monitoring of the occlusion. ${ }^{10}$ This control includes proper distribution of dental contacts, favourable deocclusion guides, elimination of premature contacts and occlusal interferences. 
Its importance is considered crucial. ${ }^{15}$

The location, number and distribution of implants are independent variables that reflect the quality and volume of bone. ${ }^{17}$ The position and the distance between implants are influenced by the wide of dental arches. ${ }^{25}$

The biomechanics and planning of prostheses will be influenced by the extent of the prosthesis, the region to rehabilitate and other factors such as the length, diameter, inclination and surface of the implants. ${ }^{31-33}$

To rehabilitate edges with little bone height, the use of short implants with standard width is not recommended. Implants with greater width can promote an adequate surface area to osseointegration providing an alternative to prosthetic support. ${ }^{32}$ The large diameter implants also promotes a better connection of the abutment screw and extends the implant platform with more occlusal support. Koyano et al evaluated three cohort studies with large samples sizes and long observation periods, and concluded that the diameter of the implants did not seem to influence implant survival rate. ${ }^{21}$

The diameter and distribution of the implants must be in accordance with the size of the occlusal platform that will be used in the prosthesis. This is decided according to the space to rehabilitate and in harmony with the remaining natural teeth. ${ }^{16,32}$

Current prosthetic designs define that the minimal number of implants to jaw is 4 while in the maxilla should be used 6 to 8 avoiding the cantilever in superior arch. ${ }^{21}$ The same protocols also require symmetrical placement of the implant in the region of the tuberosities, canines and incisors which together with a suitable occlusal scheme, support and transfer the occlusal load in a more stable manner. ${ }^{5}$

Generally the principles adopted to establish the implant-supported occlusion are used to reduce the occlusal overload on the prosthesis and protect the osseointegrated implants. ${ }^{32}$ The basic principles of implant-protected occlusion include a bilateral stability in centric occlusion, equal distribution of occlusal contacts and masticatory loads, absence of occlusal interferences, previous tab in the protrusion and whenever possible canine guide excursive movements. ${ }^{34}$ Furthermore, the adoption of procedures has been proposed to change or modify factors with cumulative effects that lead to overloading of the implants. ${ }^{23}$

The extent of occlusal table is also a possible factor for the development of occlusal overload. A wide platform increases the stress on the screws of the abutments. ${ }^{32}$ This may contraindicate the implant-supported prostheses in cases of extreme angulation and inclination of the implant where the abutments have to compensate these irregularities at the occlusal level. ${ }^{16,18,19,32}$

Cusp inclination has been found to produce a high level of torque. When cusp angle becomes greater than $15^{\circ}$, stress also increases leading to angled load to the crestal bone. ${ }^{16,19,20}$ Some authors defend that a reduced cusp inclination, shallow occlusal anatomy, and wide grooves and fossae could be beneficial for implant prosthesis. ${ }^{16,}$ 18, 19, 32 More central positioning of the implant for the future prosthesis, decreased inclination of cusps, reduction in the extent of the cantilever and obtaining a central fossa with $1.5 \mathrm{~mm}$ to keep the resulting forces in the vertical direction can lead to reduce implant overload. ${ }^{16,20,22,31}$

Prostheses with cantilevers have been used successfully in implantology. However, these must be used with caution since a large cantilever may generate an occlusal overload resulting in the peri-implant bone loss and failure of prosthetic treatment. 10,35

Sometimes for anatomical reasons it is not possible to get an ideal implant/crown proportion (1:1). ${ }^{30}$ Low bone quality and quantity in the maxillary posterior area creates the need for implant-supported prostheses with distal cantilever. A disadvantage of using a distal cantilever is the outcome of its location and intensity of masticatory forces. ${ }^{36}$ Contact must be avoided at the distal aspects of cantilever during lateral excursions. ${ }^{32}$

Cantilevers over $15 \mathrm{~mm}$ have a greater probability of failure. ${ }^{37}$ Short cantilevers promote greater success of mandibular prosthesis, especially when they are supported by small number of implants. In the upper jaw, cantilevers till $12 \mathrm{~mm}$ are also considered unfavourable. ${ }^{18,24}$ The length of cantilever should be determined by the distance between most anterior and more posterior implant. It has been proposed by Jackson et al that the cantilever length should be the value of the distance of A-P spread $\times 1.5$ (Figure I). ${ }^{32}$

Anterior or posterior contour of the prosthesis may also function as cantilever, increasing the stress in the support structures when occlusal forces are more intense. ${ }^{16,20}$ In some cases maxillar prostheses should have a cross-bite design.. This avoids the buccal cantilever and favours the axial load distribution in cases of palatal position of implants. ${ }^{19,21}$ When cantilever is associated with parafunctional activity the risk of implant overload is higher, 19, 38 Abnormal occlusal forces can contribute to prosthetic complications. ${ }^{12}$ Bruxism is a parafunction that do not contraindicate implant supported prosthetic treatment. ${ }^{9}$ However, these habits should be diagnosed and compensated when treatment finishes. In these situations, patients should use occlusal splints especially during sleep. ${ }^{9}$, $10,12,27,37$

Parafunctional activities in patients with an inadequate occlusal pattern are closely related to the peri-implant bone loss, fracture of implants and failure of prosthesis. ${ }^{19,}$ 24 The premature contacts and occlusal interferences can promote excessive lateral force with impaired osseointegration. ${ }^{18}$ Other complications related to parafunctional activities are tooth wear, crown fracture, crown de-cementation, displacement and fracture of abutment screw and peri-implant tissues trauma. 27, 39

The prognosis of oral rehabilitation in patients with parafunction is more favourable when occlusal patterns and favourable deocclusion guides are stablished and regular checks are performed through occlusal adjustments. ${ }^{10,18,37}$ Occlusal adjustment is also used to reduce the difference in mobility between natural teeth and implant-supported prosthesis. ${ }^{40}$

Several authors over the years have reported a positive relationship between the existence of parafunctional habits and peri-implant bone loss. 
Thus, it has been found that the longevity of implants subjected to high occlusal forces is related with the amount of stress and quality of bone However, the best distribution of occlusal contacts and the use of more implants can significantly reduce the burden over implants and prostheses. ${ }^{32}$ Whatever type of occlusal scheme suitable for the implant-supported prosthesis, the implant-protected occlusion principles must be complied with, to provide maximum functionality to the patient and simultaneously protect the osseointegrated implants.

\section{Clinical Guidelines}

Occlusal concepts determined in the natural dentition have been established through clinical trials and theoretical biomechanical bases, as mutually protected occlusion, bilateral balanced occlusion and deocclusion guides in lateral movements. ${ }^{2}, 8,18,23,34$

In conventional dentures or overdentures it might have bilateral contacts between the dental arches in centric or eccentric positions, and therefore, must resort to the occlusal scheme of bilateral balanced occlusion. 23,24

In cases of reabsorbed alveolar ridges in edentulous patients lingualized occlusion is recommended, where the palatal cusps of the posterior maxillary teeth occlude in the central fossa of the posterior mandibular teeth. ${ }^{23,38}$ In this occlusal pattern the upper teeth have median slope cusps and lower teeth have flat cusps, in order to eliminate the interference side during excursive movements. ${ }^{18,}$ ${ }^{40}$ In static occlusion, the goal is to match the position of centric relation with the maximum intercuspation position so that movement in the horizontal and frontal plan allows freedom of movements in centric occlusion of 1 to $1.5 \mathrm{~mm} .{ }^{16,18,41}$

These concepts have been developed in order to adapt to the oral rehabilitations with implants and as such have been proposed the following changes, distribution of occlusal contacts, platform modifications and occlusal anatomy, increase the implant surface area and adaptation of the occlusal contacts restorations with unfavourable biomechanics. 2, 16, 23 In addition, the occlusal morphology directs forces in the axial direction, the use of occlusal splints, reducing the occlusal table and the cusp inclination and reducing the extent of cantilevers are suggested factors when considering the occlusion factors applied to implantology. 2, 16, 23, 24, 34

The occlusal principles are variable depending on the planning of each case, the configuration of dental arches and prosthesis models of implants required: single, partial or total.4, 18, 23, 24,38 In Table I are summarized almost all possible clinical situations and the corresponding occlusal solutions for each case.

\section{CONCLUSIONS}

Based on the literature reviewed it can be concluded that the objective of implant-protected occlusion is to minimize occlusal overload on the implant/bone interface and on prostheses keeping the masticatory loads within physiological limits, providing stability and longevity to rehabilitation treatments with implants.

The realization of an individualized treatment plan with surgical and prosthetic procedures based on biomechanical principles is a prerequisite for successful treatment. In addition, periodic checks and occlusal adjustment made in them are essential to the success of treatment.

\section{CONFLICT OF INTEREST}

The authors declares that there is no conflict of interest regarding the publication of this article. 


\section{ATTACHMENT}

\begin{tabular}{|c|c|}
\hline Clinical situation & Occlusion Principles \\
\hline $\begin{array}{l}\text { Implant-supported fixed } \\
\text { prosthesis }\end{array}$ & $\begin{array}{l}\text { Bilateral balanced occlusion when the antagonist is a conventional full denture } \\
\text { Group function or occlusion mutually protected antagonist on natural teeth } \\
\text { Infraocclusion below the cantilevered segment } \\
\text { Freedom movement in centric movements ( } 1 \text { a } 1,5 \mathrm{~mm} \text { central fossa) }\end{array}$ \\
\hline $\begin{array}{l}\text { Implant-supported } \\
\text { overdentures }\end{array}$ & $\begin{array}{l}\text { Bilateral balanced occlusion } \\
\text { Lingualized or monoplane Occlusion }\end{array}$ \\
\hline $\begin{array}{l}\text { Posterior implant-supported } \\
\text { prosthesis }\end{array}$ & $\begin{array}{l}\text { Anterior guidance in natural teeth } \\
\text { Group function to compromised canines } \\
\text { Centralized contacts, occlusal table and reduced cusps tilt and cantilever reduction } \\
\text { Posterior crossbite } \\
\text { Tooth wear natural teeth in case of periodontal disease }\end{array}$ \\
\hline $\begin{array}{l}\text { Unitary implant-supported } \\
\text { prosthesis }\end{array}$ & $\begin{array}{l}\text { Anterior guidance in natural teeth } \\
\text { Light bites and strong contacts in the absence of contacts in light bites } \\
\text { centralized contacts } \\
\text { Increasing proximal contacts } \\
\text { Elimination of dental interferences during eccentric movements }\end{array}$ \\
\hline Poor bone quality & $\begin{array}{l}\text { Increase healing time } \\
\text { Gradual increase of occlusal loading }\end{array}$ \\
\hline
\end{tabular}

Table 1. Summary of clinical applicability of occlusal schemes in implantology

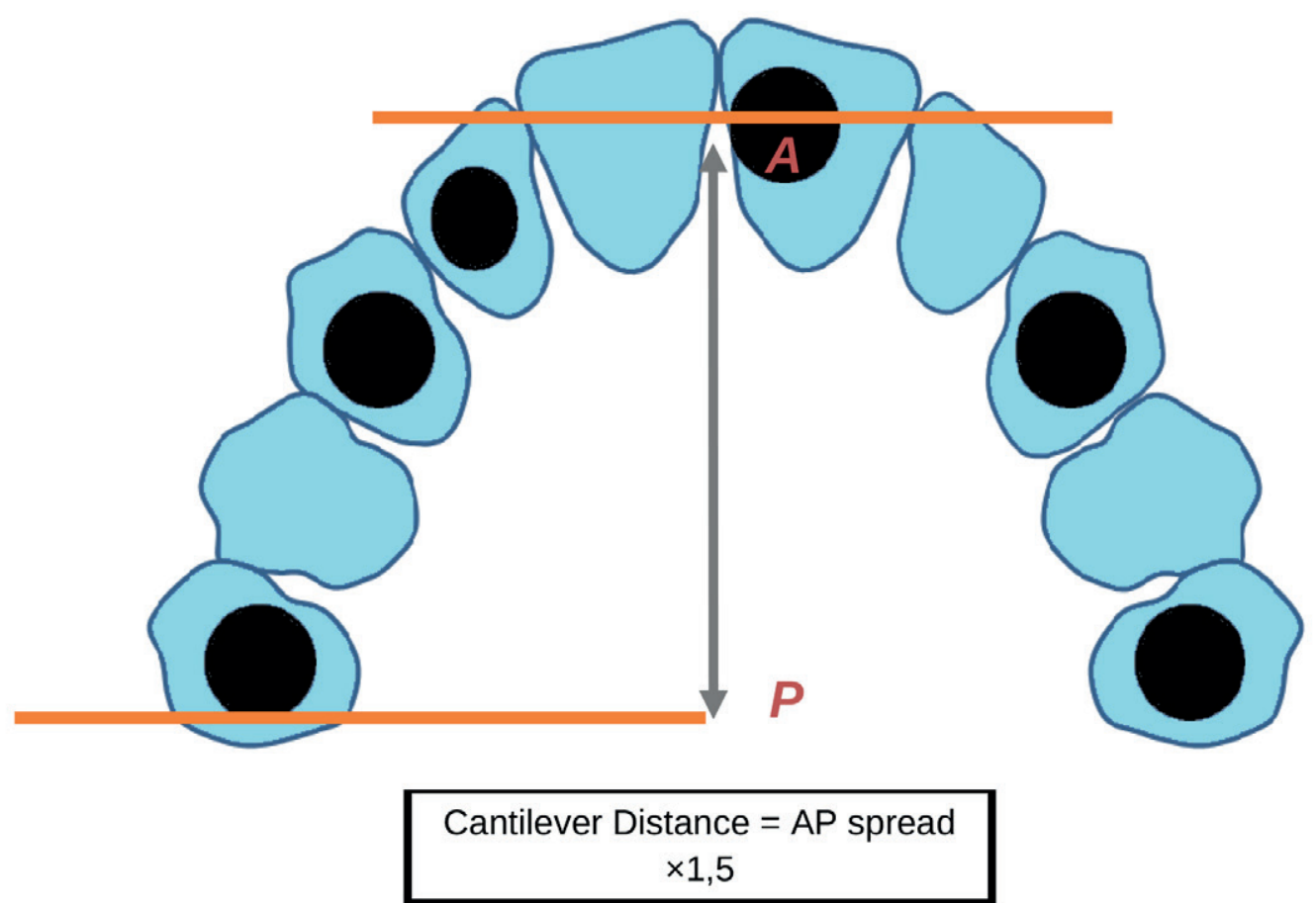

Figure 1. Protocol to predict the most favourable length of the prosthetic cantilever 


\section{REFERENCES}

1. Chang M, Chronopoulos V, Mattheos N. Impact of excessive occlusal load on successfully-osseointegrated dental implants: a literature review. Journal of investigative and clinical dentistry. 2013;4(3):142-50.

2. Tiwari B, Ladha K, Lalit A, Dwarakananda Naik B. Occlusal concepts in full mouth rehabilitation: an overview. Journal of Indian Prosthodontic Society. 2014;14(4):344-51.

3. Naert I, DuyckJ, Vandamme K. Occlusal overload and bone/implant loss. Clinical oral implants research. 2012;23 Suppl 6:95-107.

4. Rilo B, da Silva JL, Mora MJ, Santana U. Guidelines for occlusion strategy in implant-borne prostheses. A review. International dental journal. 2008;58(3):139-45.

5. Duyck J, Naert I. Failure of oral implants: aetiology, symptoms and influencing factors. Clinical oral investigations. 1998;2(3):102-14.

6. Berglundh T, Persson L, Klinge B. A systematic review of the incidence of biological and technical complications in implant dentistry reported in prospective longitudinal studies of at least 5 years. Journal of clinical periodontology. 2002;29 Suppl 3:197-212; discussion 32-3.

7. Merin RL. Repair of peri-implant bone loss after occlusal adjustment: a case report. Journal of the American Dental Association (1939). 2014;145(10):1058-62.

8. Duyck J, Vandamme K. The effect of loading on peri-implant bone: a critical review of the literature. Journal of oral rehabilitation. 2014;41(10):78394.

9. Lobbezoo F, Van Der Zaag J, Naeije M. Bruxism: its multiple causes and its effects on dental implants - an updated review. Journal of oral rehabilitation. 2006;33(4):293-300.

10. Manfredini D, Bucci MB, Sabattini VB, Lobbezoo F. Bruxism: overview of current knowledge and suggestions for dental implants planning. Cranio : the journal of craniomandibular practice. 2011;29(4):304-12.

11. Todescan S, Lavigne S, Kelekis-Cholakis A. Guidance for the maintenance care of dental implants: clinical review. Journal (Canadian Dental Association). 2012;78:c107.

12. Johansson A, Omar R, Carlsson GE. Bruxism and prosthetic treatment: a critical review. Journal of prosthodontic research. 2011;55(3):127-36.

13. 1 Meyer G, Fanghanel J, Proff P. Morphofunctional aspects of dental implants. Annals of anatomy= Anatomischer Anzeiger : official organ of the Anatomische Gesellschaft. 2012;194(2):190-4.

14. Conrad HJ, Schulte JK, Vallee MC. Fractures related to occlusal overload with single posterior implants: a clinical report. The Journal of prosthetic dentistry. 2008;99(4):251-6.

15. Ghoul WE, Chidiac JJ. Prosthetic requirements for immediate implant loading: a review. Journal of prosthodontics : official journal of the American College of Prosthodontists. 2012;21(2):141-54.

16. Klineberg I, Kingston D, Murray G. The bases for using a particular occlusal design in tooth and implant-borne reconstructions and complete dentures. Clinical oral implants research. 2007;18 Suppl 3:151-67.

17. Boyce RA, Klemons G. Treatment planning for restorative implantology. Dental clinics of North America. 2015;59(2):291-304.

18. Kim Y, Oh TJ, Misch CE, Wang HL. Occlusal considerations in implant therapy: clinical guidelines with biomechanical rationale. Clinical oral implants research. 2005;16(1):26-35.

19. Chen Y, Kuan C, Wang Y. Implant occlusion: biomechanical considerations for implant-supported prostheses. J Dent Sci. 2008;3(2):65-74.

20. Swaminathan Y, Rao G. Implant protected occlusion. IOSR-JDMS. 2013;11(3):20-5.

21. Koyano K, Esaki D. Occlusion on oral implants: current clinical guidelines. Journal of oral rehabilitation. 2015;42(2):153-61.

22. Weinberg LA. Therapeutic biomechanics concepts and clinical procedures to reduce implant loading. Part I. The Journal of oral implantology 2001;27(6):293-301.

23. Abichandani S, Bhojaraju N, Guttal S, Srilakshmi J. Implant protected occlusion: a comprehensive review. Eur J Prosthodont. 2013;1(2):29-36.

24. Dhanasekar B, Aparna I, Neha M, Amit G. Occlusion in implant dentistry - Issues and considerations. .J Oral Health Comm Dent 2012;6(2):91-6.

25. Gross MD. Occlusion in implant dentistry. A review of the literature of prosthetic determinants and current concepts. Australian dental journal. 2008;53 Suppl 1:S60-8.

26. Eskitascioglu G, Usumez A, Sevimay M, Soykan E, Unsal E. The influence of occlusal loading location on stresses transferred to implant-supported prostheses and supporting bone: A three-dimensional finite element study. The Journal of prosthetic dentistry. 2004;91(2):144-50.

27. Komiyama O, Lobbezoo F, De Laat A, lida T, Kitagawa T, Murakami H, et al. Clinical management of implant prostheses in patients with bruxism. International journal of biomaterials. 2012;2012:369063.

28. Palma-Carrio C, Maestre-Ferrin L, Penarrocha-Oltra D, Penarrocha-Diago MA, Penarrocha-Diago M. Risk factors associated with early failure of dental implants. A literature review. Medicina oral, patologia oral y cirugia bucal. 2011;16(4):e514-7.

29. Miyata T, Kobayashi Y, Araki H, Ohto T, Shin K. The influence of controlled occlusal overload on peri-implant tissue. part 4: a histologic study in monkeys. The International journal of oral \& maxillofacial implants. 2002;17(3):384-90.

30. Isidor F. Influence of forces on peri-implant bone. Clinical oral implants research. 2006;17 Suppl 2:8-18.

31. Sanchez-Perez A, Moya-Villaescusa MJ, Jornet-Garcia A, Gomez S. Etiology, risk factors and management of implant fractures. Medicina oral, patologia oral y cirugia bucal. 2010;15(3):e504-8

32. Jackson BJ. Occlusal principles and clinical applications for endosseous implants. The Journal of oral implantology. 2003;29(5):230-4.

33. Hasan I, Bourauel C, Mundt T, Stark H, Heinemann F. Biomechanics and load resistance of small-diameter and mini dental implants: a review of literature. Biomedizinische Technik Biomedical engineering. 2014;59(1):1-5.

34. Jambhekar S, Kheur M, Khothavade M, Dugal R. Occlusion and occlusal considerations in Implantology. IJDA. 2010;2(1):125-30.

35. Taruna M, Chittaranjan B, Sudheer N, Tella S, Abusaad M. Prosthodontic perspective to all-on-4(R) concept for dental implants. Journal of clinical and diagnostic research : JCDR. 2014;8(10):Ze16-9.

36. Quirynen T, Quirynen M, DuyckJ. Prevention of distal extension cantilever fracture in mandibular overdentures. Journal of dentistry. 2015;43(9):1140-7

37. Salvi GE, Bragger U. Mechanical and technical risks in implant therapy. The International journal of oral \& maxillofacial implants. 2009;24 Suppl:69-85.

38. Pita M, Anchieta R, Pita D, Zuim P, Pellizzer E. Occlusion rationales in implantology: clinical guidelines and their prosthetic and biomechanics determinants. Rev odontol UNESP. 2008;29(1):53-9.

39. Kinsel RP, Lin D. Retrospective analysis of porcelain failures of metal ceramic crowns and fixed partial dentures supported by 729 implants in 152 patients: patient-specific and implant-specific predictors of ceramic failure. The Journal of prosthetic dentistry. 2009;101(6):388-94.

40. Lundgren D, Laurell L. Biomechanical aspects of fixed bridgework supported by natural teeth and endosseous implants. Periodontology 2000 $1994 ; 4: 23-40$

41. Wiens J, Priebe J. Occlusal Stability. Dent Clin N Am. 2014;58(1):19-43. 